\title{
The level of myeloid-derived suppressor cells positively correlates with regulatory $T$ cells in the blood of children with transient hypogammaglobulinaemia of infancy
}

\author{
IZABELA SIEMIŃSKA ${ }^{2 *}$, MAGDALENA RUTKOWSKA-ZAPAEA ${ }^{I^{*}}$, \\ KAROLINA BUKOWSKA-STRAKOVA ${ }^{l}$, ANNA GRUCA ${ }^{l}$, ANNA SZAFLARSKA ${ }^{l}$, \\ KRZYSZTOF KOBYLARZ ${ }^{2}$, MACIEJ SIEDLAR ${ }^{l}$, JAREK BARAN ${ }^{l}$
}

${ }^{1}$ Department of Clinical Immunology, Institute of Paediatrics, Faculty of Medicine, Jagiellonian University Medical College, Krakow, Poland ${ }^{2}$ Department of Anaesthesiology and Intensive Care, Institute of Paediatrics, Faculty of Medicine, Jagiellonian University Medical College, Krakow, Poland

"These authors contributed equally to this work.

\begin{abstract}
Introduction: Transient hypogammaglobulinaemia of infancy (THI) is a primary immunodeficiency characterised by low levels of immunoglobulin G (often with concomitant decrease of IgA and sometimes also of IgM) with still unknown exact reason. A delayed normalisation of the immunoglobulin level in THI may be associated with a transiently elevated number of regulatory T cells (Treg). Although in cancer and chronic inflammation it was shown that the level of Treg cells can be increased by myeloid-derived suppressor cells (MDSCs), until now no studies have been performed in the context of the role of MDSCs in THI and their correlation with Treg cells. Consequently, we aimed to determine the occurrence of MDSCs in the peripheral blood of children with THI and correlate their level with the level of Treg cells.

Material and methods: Flow cytometry analyses of Mo-MDSCs and Gr-MDSCs, characterised as $H L A-D R^{-} C D 11 b^{+} C D 15^{-} C D 14^{+}$and $H L A-D R^{-} C D 11 b^{+} C D 15^{+} C D 14^{-}$, respectively, and Treg $\left(C D 4^{+} C-\right.$ $D 25^{+} \mathrm{Foxp}^{+}$) cells were performed.

Results: The proportion of Mo-MDSCs and Gr-MDSCs was significantly higher in the group of THI patients with elevated level of Treg cells (from the 95\% confidence interval level of healthy controls). The cells with Mo-MDSC and Gr-MDSC characteristics positively correlated with the level of Treg cells. Moreover, children with a higher proportion of circulating Treg cells, and thereby higher level of MDSCs, showed delayed normalisation of IgG level and recovery.

Conclusions: These findings show for the first time that MDSCs may be involved in the pathomechanism of THI, probably acting through the induction of Treg cells.
\end{abstract}

Key words: MDSCs, regulatory T cells, THI.

(Centr Eur J Immunol 2018; 43 (4): 413-420)

\section{Introduction}

Myeloid-derived suppressor cells (MDSCs) compose a heterogeneous population of immature myeloid progenitors with immunosuppressive properties [1, 2]. It has been demonstrated that the level of MDSCs increases in chronic inflammatory conditions, infections, and cancer, contrary to healthy individuals, where MDSCs represent a scant percentage $[1,3]$. Several studies have shown that human MDSCs are phenotypically characterised as neg- ative for linear markers CD3 and CD19, low expression of HLA-DR, and high expression of CD33 and CD11b. In peripheral blood MDSCs are divided into two main subpopulations: monocytic (Mo-MDSCs) and granulocytic (Gr-MDSCs), identified as CD $11 \mathrm{~b}^{+} \mathrm{CD} 33^{+} \mathrm{HLA}^{-D R}{ }^{-}$ CD $14^{+} \mathrm{CD} 15^{-}$and $\mathrm{CD} 11 \mathrm{~b}^{+} \mathrm{CD} 33^{+} \mathrm{HLA}^{-\mathrm{DR}}{ }^{-\mathrm{CD}} 14^{-} \mathrm{CD} 15^{+}$, respectively, as recommended by the Mye-EUNITER consortium [3, 4]. It has been shown that MDSC-mediated immunosuppression involves multiple mechanisms,

Correspondence: Jarek Baran, PhD, DSc, Department of Clinical Immunology, Institute of Paediatrics, Faculty of Medicine,

Jagiellonian University Medical College, 265 Wielicka St., 30-663 Krakow, Poland, e-mail: mibaran@cyf-kr.edu.pl

Submitted: 28.06.2018; Accepted: 4.12.2018 
such as inhibition of adaptive immune response mediated by $\mathrm{CD}^{+}$and $\mathrm{CD}^{+} \mathrm{T}$ cells $[5,6]$ as well as induction of the immunosuppressive population of regulatory $\mathrm{T}$ (Treg) cells [7]. Moreover, crosstalk between Treg cells and MDSCs has been clearly documented [7, 8], and the main factors released by MDSCs involved in the induction of Treg cells have been identified as: IL-10, transforming growth factor $\beta$ (TGF- $\beta$ ), enzymes - arginase- 1 (ARG1) (E.C. 3.5.3.1), and inducible nitric oxide synthase (NOS2) (EC 1.14.13.39), which reduce the concentration of L-arginine in the tissue milieu [9]. Furthermore, NOS2 and NADPH oxidase have been shown to be responsible for upregulation of reactive nitrogen species (RNS) and reactive oxygen species (ROS). These two mechanisms cause inhibition of T-cell proliferation by blocking CD3 zeta chain expression [10, 11]. Gr-MDSCs mainly generate ROS, whereas Mo-MDSCs produce higher levels of RNS [12]. The longer half time of NO than ROS determines that Mo-MDSCs are considered to be more suppressive than Gr-MDSCs and do not need direct cell-to-cell contact to maintain their suppressive functions [13, 14].

Although MDSCs were initially identified and characterised as a new population that plays an important role in cancer progression $[15,16]$, the list of conditions in which MDSCs are relevant is still getting longer. Over the last few years an increasing number of reports have described their role in viral infections [17], bacterial infections [18], parasitic infections [19], traumatic stress [20], and conditions after organ transplantation [21]. However, there are no data showing the potential role of MDSCs in the pathogenesis of any primary immunodeficiency disease (PIDs).

Predominantly, immunoglobulin deficiencies are the most common forms of PIDs [22, 23]. One of them, transient hypogammaglobulinaemia of infancy (THI), is a heterogeneous disorder characterised by reduced serum IgG (often IgA and sometimes also IgM) level in early childhood and recurrent infections, mostly of the respiratory tract [24-26]. A putative diagnosis is initially made after exclusion of other causes of hypogammaglobulinaemia, while a definitive diagnosis of THI can only be made retrospectively in patients with normalised $\mathrm{IgG}$ levels and withdrawal of clinical symptoms, which occurs usually between the second and fourth year of life [27]. The underlying definitive basis of this disorder is still unknown. Based on the latest reports, a possible role of a transiently elevated number of Treg cells in THI patients was considered. It was shown that the initially elevated Treg cells in patients with THI decreases with age, reaching at the time of recovery the levels observed in healthy subjects. Decrease of Treg cells in the THI group was associated with normalisation of immunoglobulin level and withdrawal of clinical symptoms [28]. Furthermore, inhibitory effects of Treg cells on B-cell functions and immunoglobulin production were described [29, 30]. Thus, it was interesting to analyse whether the level of MDSCs in peripheral blood of THI patients is elevated at the time of initial diagnosis and correlates with the proportion of circulating Treg cells.

\section{Material and methods}

\section{Patients}

The studied group was selected from patients referred to the outpatient clinic of the Department of Clinical Immunology, University Children's Hospital in Krakow, Poland. All patients were diagnosed according to criteria of the International Union of Immunological Societies [31]. Sixteen patients (13 boys and 3 girls), whose levels of immunoglobulins normalised with age, were retrospectively classified as THI. Such a gender distribution was a consequence of including consecutive patients from the outpatient clinic. The mean age of patients at the time of initial diagnosis was $9.2 \pm 7.06$ months. Children in whom immunodeficiency diseases were excluded $(n=6)$, formed gender (five boys and one girl) and an age-matched control group. Written, informed consent was obtained from the legal representatives of the patients. The Bioethical Committee of Jagiellonian University approved the study (no. 122.6120.195.2015). Determination of the level of MDSCs and Treg cells was made at the time of initial diagnosis and considered for analysis after retrospective verification.

\section{Isolation of peripheral blood mononuclear cells}

Whole peripheral blood samples from patients and healthy control subjects were drawn to EDTA-containing tubes (Vacutainer System; Becton Dickinson, San Jose, CA). Peripheral blood mononuclear cells (PBMC) were isolated by the standard Ficoll-Paque (Pharmacia, Uppsala, Sweden) density gradient centrifugation.

\section{Determination of Mo-MDSC and Gr-MDSC levels}

For MDSCs analysis, PBMC (app. $1 \times 10^{6}$ cells) were stained with the following monoclonal antibodies (mAbs): anti-HLA-DR-PerCP (clone L243), anti-CD11b-BV510 (clone ICR F44), anti-CD14-FITC (clone M $\phi P 9)$, and anti-CD15-PE-Cy7 (clone HI98) (all from Pharmingen, BD Biosciences, San Diego, CA) for $20 \mathrm{~min}$ in $4^{\circ} \mathrm{C}$. After incubation, cells were washed twice in PBS and suspended in $0.2 \mathrm{ml}$ PBS. In order to determine the level of non-specific staining and autofluorescence, fluorescence minus one (FMO) control samples were incubated in parallel. The samples were analysed in a FACSCanto flow cytometer (BD Biosciences, Immunocytometry systems, San Jose, CA) using FACSDiva software (BD Biosciences). The Mo-MDSCs were characterised as HLA-DR-CD$11 \mathrm{~b}^{+} \mathrm{CD} 14^{+} \mathrm{CD} 15^{-}$cells and presented as the percentage of nucleated cells (NC) (positive for SYTO ${ }^{\text {TM }} 9$ staining; Invitrogen, Eugene, OR), whereas Gr-MDSCs, like HLA- 

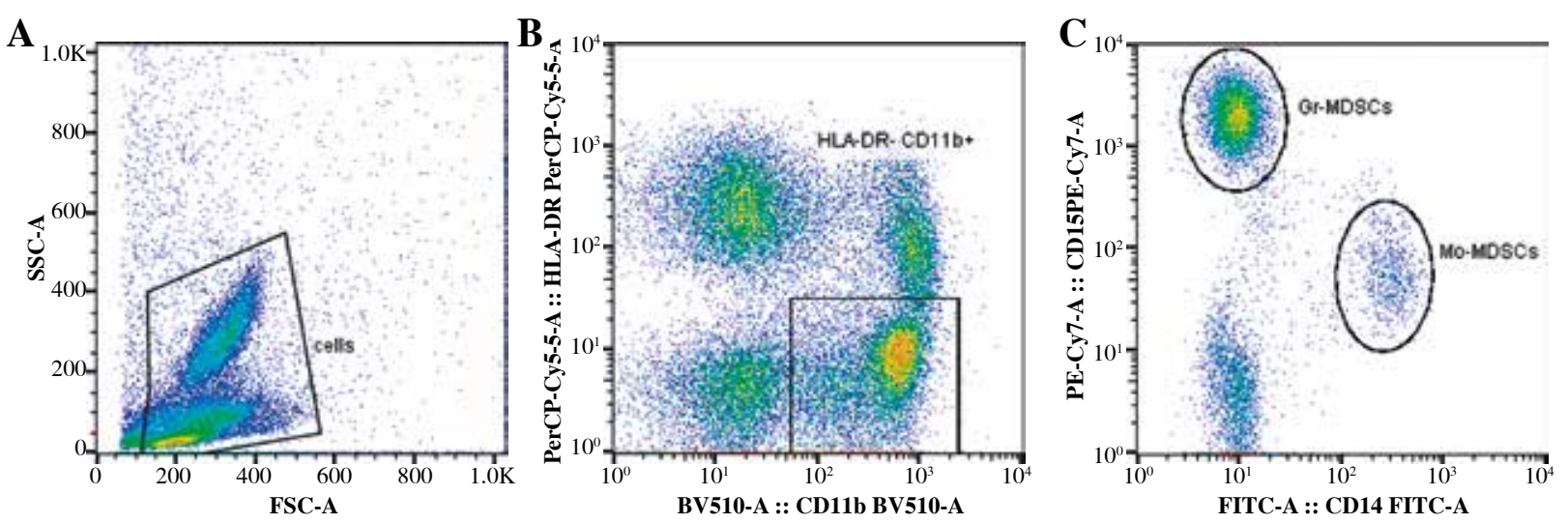

Fig. 1. Gating strategy for flow cytometry identification of Mo-MDSCs and Gr-MDSCs in the peripheral blood of THI patients

$\mathrm{DR}^{-} \mathrm{CD} 11 \mathrm{~b}^{+} \mathrm{CD} 14^{-} \mathrm{CD} 15^{+}$cells, were presented also as a percentage of NC (Fig. 1).

\section{Determination of Treg cell levels}

For Tregs cell analysis, PBMCs were stained using a Human Regulatory T-cell Staining Kit (BD Biosciences) with the following mAbs: anti-CD4-FITC, anti-CD25$\mathrm{PE}$, and anti-Foxp3-APC, according to the manufacturer's instructions. In parallel, a control sample was prepared to determine the non-specific binding of antibodies (isotype control). Samples were analysed in a FACSCanto flow cytometer using FACSDiva software. The level of Treg cells was calculated as the proportion of $\mathrm{CD} 4^{+} \mathrm{CD} 25^{+} \mathrm{Foxp} 3^{+}$ cells from the whole $\mathrm{CD} 4^{+}$population.

\section{Assessment of the immunoglobulin level}

Concentrations of serum immunoglobulins $\operatorname{IgG}$ and IgA were determined by nephelometry method (BNII nephelometer; Dade-Behring, Deerfield, IL; antisera from Behring) during routine laboratory tests.

\section{Statistical analysis}

Statistical analysis was performed using the PRISM GraphPad 5 package (GraphPad Software Inc., San Diego, CA, USA). Obtained data were analysed using t-test or one-way analysis of variance (ANOVA) with Dunnett's Multiple Comparison Test, as a post hoc test. The magnitude of the relationship between two quantitative features was evaluated using Pearson's correlation coefficient. Normality before t- test was tested by Shapiro-Wilk test, and for non-parametric data U-Mann-Whitney test was used (comparison of Mo-MDSC and Gr-MDSC levels in THI and healthy controls). All data are expressed as mean $\pm \mathrm{SD}$. The level $p<0.05$ was considered statistically significant.

\section{Results}

\section{Treg cells in THI patients and healthy controls}

The patient group was composed of 16 children (mean age at the time of initial diagnosis was $9.2 \pm 7.06$ months), in whom, retrospectively, THI was diagnosed, according to criteria of the International Union of Immunological Societies. There was no statistical difference in the Treg cell level between THI patients and healthy controls (CTR) $(1.53 \% \pm 0.75 \%$ in CTR vs. $3.33 \% \pm 2.40 \%$ in THI, $p=$ 0.0919) (Fig. 2A). Because our THI group was highly heterogeneous in respect to Treg cell level, it was somewhat arbitrarily divided into two subgroups, as we did previously [28]. The first group, named "THI low", consisted of seven patients with Treg cell level comparable to healthy controls, whereas in the second group, containing nine patients and named "THI high", the Treg cell level was from the $95 \%$ confidence interval level of the healthy control (0.75-2.32\% of Foxp $3^{+}$from CD4 ${ }^{+}$cells). We applied such an arbitrary division of the patients' group due to the small number of patients included and the inability to apply another type of analysis, e.g. cluster analysis, based on our previous studies [28]. The proportion of Treg cells in the "THI high" group was found to be significantly higher than in healthy controls $(1.53 \% \pm 0.75 \%$ in CTR vs. $5.06 \% \pm 1.67 \%$ in "THI high", $p<0.05$ ), as well as in the "THI low" group $(5.06 \% \pm 1.67 \%$ in "THI high" vs. $1.09 \%$ $\pm 0.56 \%$ in "THI low", $p<0.001$ ) (Fig. 2 B).

\section{Mo-MDSCs and Gr-MDSCs levels in THI patients and healthy controls}

In the next step, the proportions of Mo-MDSCs and Gr-MDSCs in peripheral blood of THI patients were analysed and compared with healthy controls. There was no statistical difference between the calculated mean percent- 

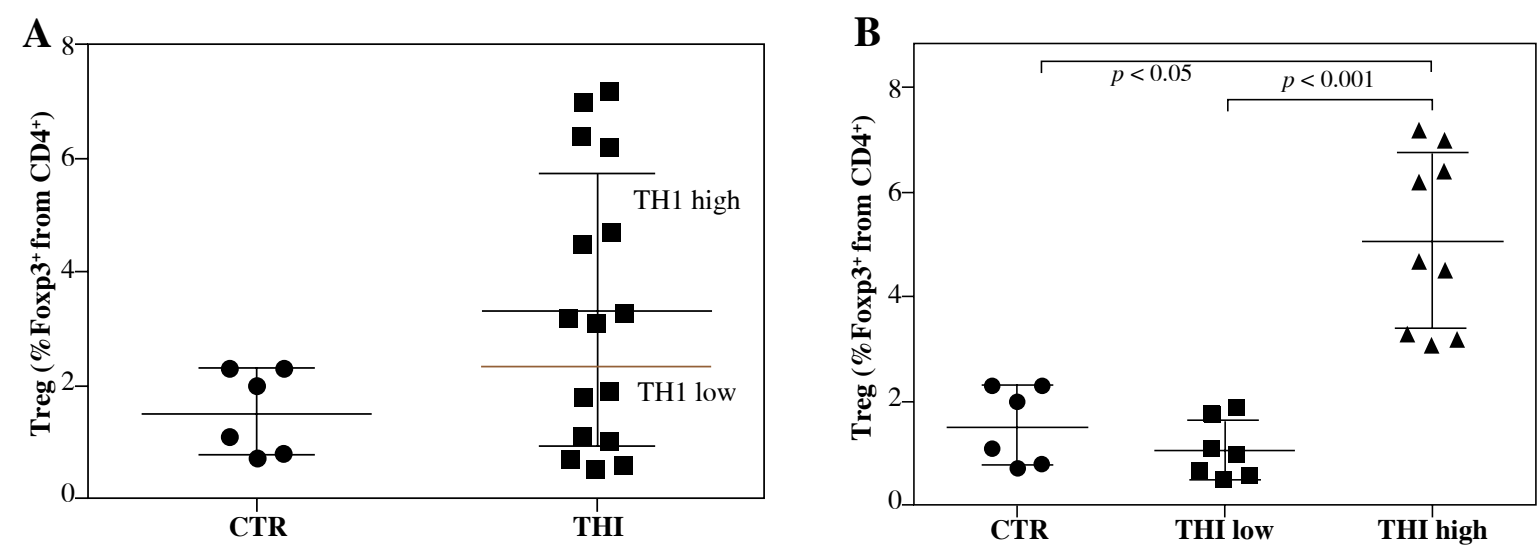

Fig. 2. Treg cell levels in THI patients and healthy age-matched control subjects (A), red line indicates the border dividing the THI patients into two subgroups. Groups of THI patients with a high and a low percentage of Treg cells, in comparison to healthy control subjects $(\mathbf{B})$
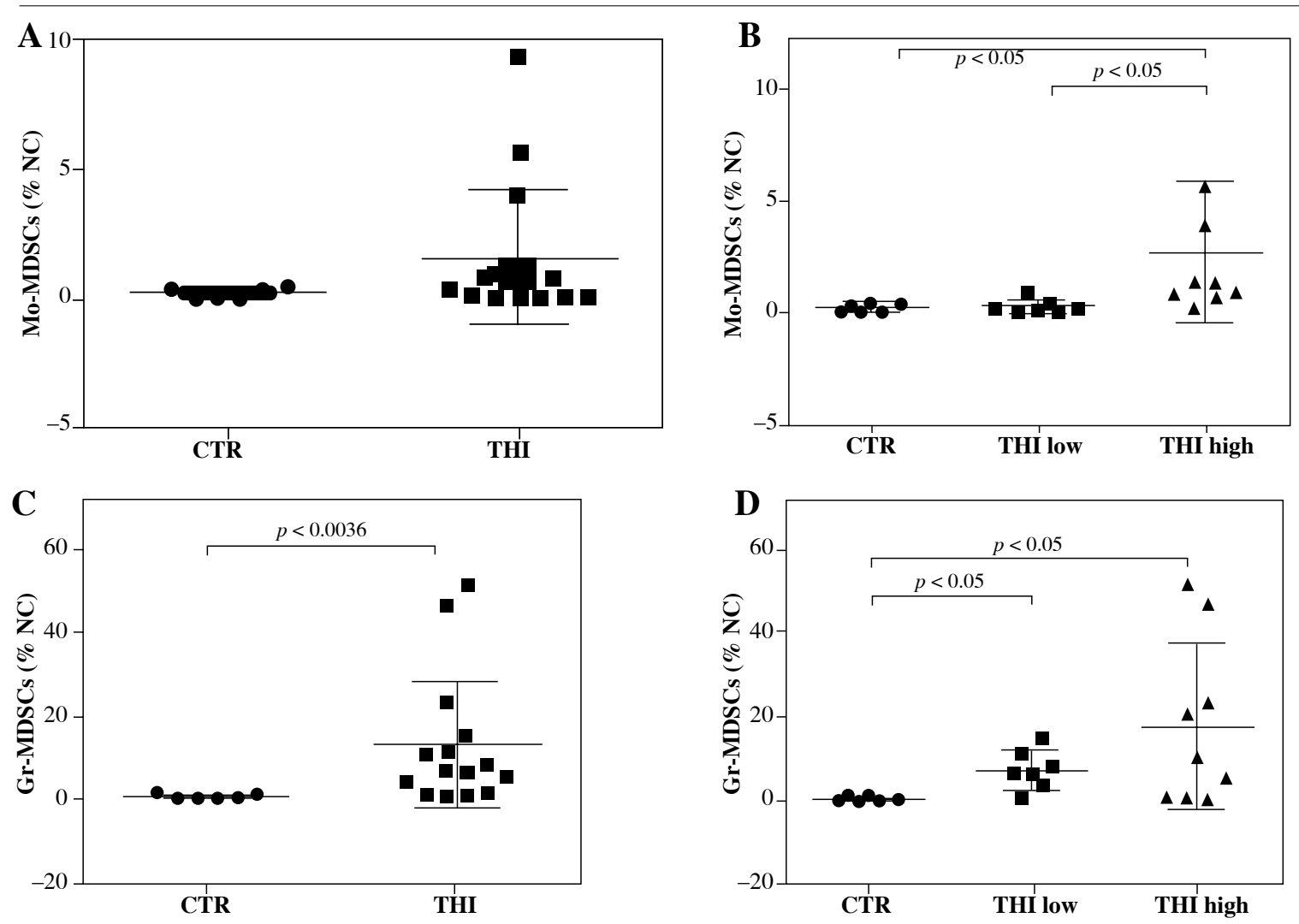

Fig. 3. Mo-MDSCs (A) and Gr-MDSCs (C) percentage in THI patients and healthy control subjects, Mo-MDSCs (B), and Gr-MDSCs (D) percentage in THI groups with a high and low levels of Treg cells, in comparison to control group (CTR) (\% NC - \% of nucleated cells)

age value of Mo-MDSCs in the group of THI patients and controls $(0.27 \% \pm 0.18 \%$ in CTR vs. $1.66 \% \pm 2.58 \%$ in THI, $p=0.0676$ ) (Fig. 3A). However, it reached statistical significance when referring to the "THI high" group, compared to the controls $(0.27 \% \pm 0.18 \%$ in CTR vs. $2.71 \%$ $\pm 3.09 \%$ in "THI high", $p<0.05$ ) or to the "THI low" group of children $(2.71 \% \pm 3.09 \%$ in "THI high" vs. $0.30 \%$ $\pm 0.30 \%$ in "THI low", $p<0.05$ ) (Fig. 3B). In respect to the second population of MDSCs, the mean percentage value of Gr-MDSCs in peripheral blood was significantly higher in THI patients in comparison to the control group $(0.58 \%$ $\pm 0.53 \%$ in CTR vs. $13.17 \% \pm 15.54 \%$ in THI, $p=0.0036$ ) 

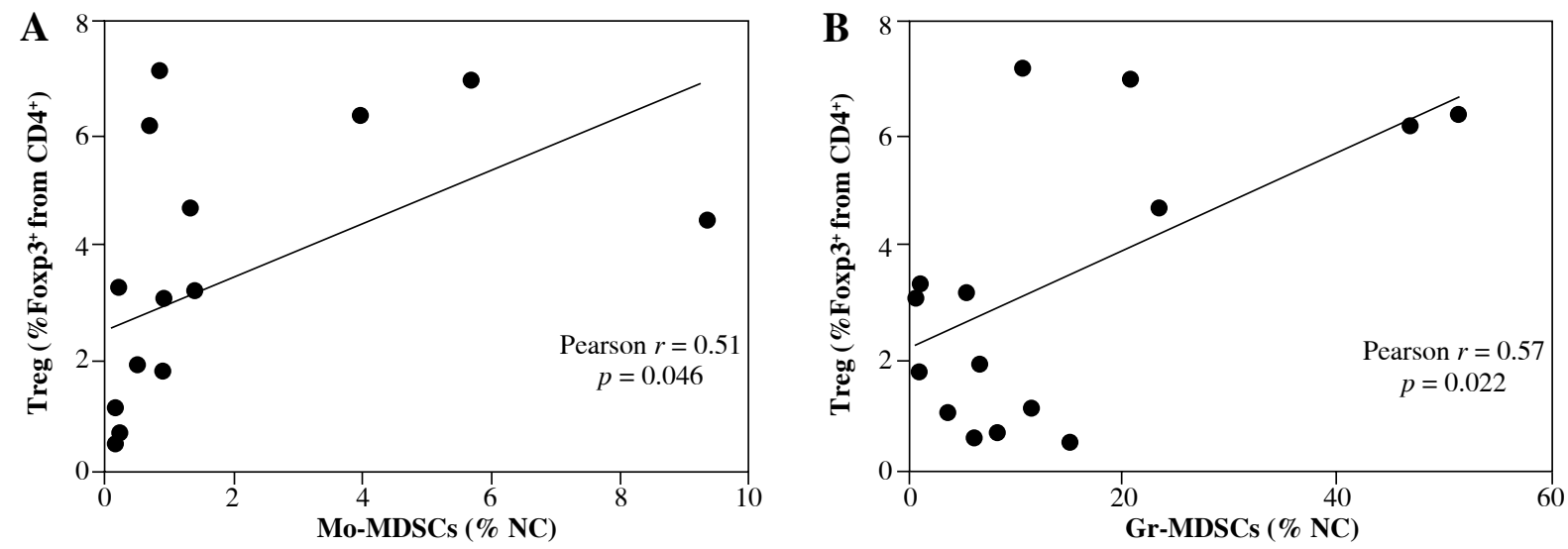

Fig. 4. The relationship between the percentage of Mo-MDSCs (A) and Gr-MDSCs (B) and the level of Treg cells in peripheral blood of children with THI

(Fig. 3C). Similarly, as was done for Mo-MDSCs, the level of Gr-MDSCs was calculated also in the two selected subgroups of THI patients. Accordingly, the mean percentage value of Gr-MDSCs in the "THI high" group was significantly elevated, compared to controls $(0.58 \% \pm 0.53 \%$ in CTR vs. $17.73 \% \pm 19.57 \%$ in "THI high", $p<0.05)$ and THI children with a low level of Treg cells $(7.73 \%$ $\pm 19.57 \%$ in "THI high" vs. $7.30 \% \pm 4.67 \%$ in "THI low", $p<0.05$ ) (Fig. 3D).

\section{Correlation between the level of Treg cells and MDSCs}

Next, we asked whether any correlation exists between the level of Treg cells and MDSCs in the blood of THI patients. The obtained results indicated a statistically significant positive correlation between the level of Treg cells and proportions of cells with Mo-MDSCs as well as Gr-MDSCs phenotype (Fig. 4). Correlation with MDSCs and Treg cells in THI patients indicates that the "THI low" group consists of patients with lower (than controls) levels of Treg cells, Mo-MDSCs, and Gr-MDSCs, whereas patients with higher (than controls) levels of these cell populations have been included into the "THI high" group. There was no correlation between the cumulative level of both MDSC populations and Treg cells in THI patients (data not shown).

\section{Time required for IgG normalisation in THI groups}

Finally, it was interesting to ask whether there were differences in the duration required for IgG normalisation, in respect to the level of circulating Treg and MDSCs at the time of initial diagnosis. This correlation was analysed in the "THI low" and "THI high" groups of patients. The obtained results indicated that $\operatorname{IgG}$ level normalised significantly more slowly in the group of children with high levels of Treg, Mo-MDSCs, and Gr-MDSCs (“THI high") compared to the group with low Treg, Mo-MDSC, and Gr-MDSC proportions ("THI low") (25.56 \pm 9.13 months in "THI high" vs. $14.57 \pm 4.24$ months in "THI low", $p=$ 0.0109) (Fig. 5).

\section{Discussion}

The strong regulatory effects of MDSCs have been reported in various clinical conditions, including cancer, infections, chronic inflammation, autoimmunity, and status post organ transplantation [17-21]. Accumulated data has already confirmed the immunosuppressive nature of MDSCs by documenting their ability to inhibit effector T cell [32] or macrophage [33] activity, as well as induction of Treg cells [34]. However, up to now the association of MDSCs with the level of Treg cells has not been analysed in PIDs.

Previously, we reported a possible role of Treg cells in the pathomechanism of THI, where elevated levels of

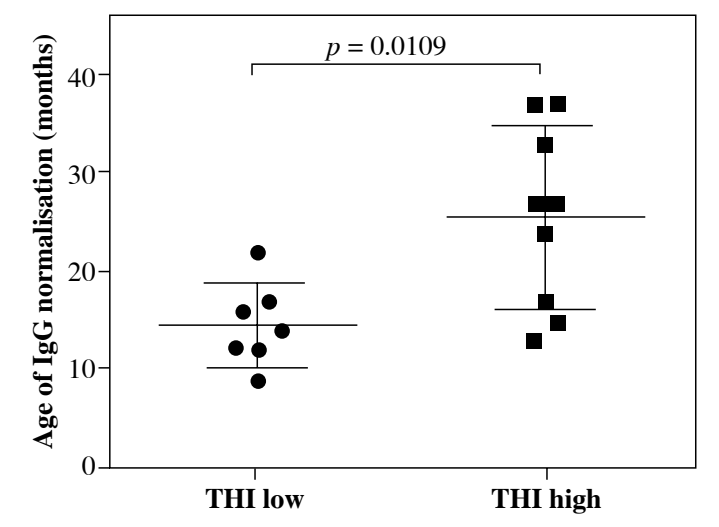

Fig. 5. Time required for normalisation of $\mathrm{IgG}$ level in THI group with high and low levels of Treg cells 
Treg cells were associated with hypogammaglobulinaemia $[28,29]$. Based on this, in the current study, we aimed to analyse the level of Mo-MDSC and Gr-MDSC populations in the peripheral blood of THI patients and check whether it correlates with the level of Treg cells at the time of initial diagnosis.

Our findings demonstrate that in the THI group with a higher proportion of Treg cells, the level of MDSCs was also significantly higher. Conversely, in THI patients with a low proportion of circulating Treg cells, the level of Mo-MDSCs was comparable to healthy control subjects, whereas the level of Gr-MDSCs was higher. These observations suggest that MDSCs in THI patients may induce Treg cells that directly inhibit IgG production by B lymphocytes [35]. A positive correlation between MDSCs and Treg cells was previously described mainly in cancer patients [36, 37], where MDSCs could induce Treg cells by IL-10 and TGF- $\beta$ production [37]. From the other hand, Treg cells can enhance MDSCs function and control their differentiation through a mechanism involving TGF- $\beta$, because TGF- $\beta$-deficient Treg cells were not able to regulate MDSCs function in an experimentally induced model of colitis [38]. This indicates a positive feedback loop established between these two cell types [38]. It is worth pointing out that in our study the proportion of Gr-MDSCs better correlated with Treg cells than the level of Mo-MDSCs did, which could suggest that the Gr-MDSCs population is mainly involved in inhibition of immunoglobulin production, confirming their suppressive role on B lymphocytes [39].

The mechanism underlying MDSC activation in THI patients remains unclear. Nevertheless, we assume that the factors similar to those seen in cancer patients could be involved in this process [40], especially that the elevated levels of tumour necrosis factor $\alpha$ (TNF- $\alpha)$ and IL-10 have been shown previously in the serum of THI patients [41].
Additionally, analysis of the relationship between the age of the patients and their IgG level has shown a positive correlation up to the normalisation of $\mathrm{IgG}$, as confirmed by the previous studies [28]. Therefore, we checked if the level of Treg cells has any impact on the duration required for IgG normalisation in THI patients. For this purpose, we compared the age of patients with normalised IgG level from the group to those with a low and high Treg cell level: "THI high" and "THI low", respectively. We noticed that in the group with a high level of Treg cells, and thereby also MDSCs, the patients did normalise their IgG level at the mean age of 26 months, while in the group with a low proportion of Treg, all patients aligned the IgG concentrations to the normal range almost one year earlier on average (Fig. 5).

In THI patients with a low number of Treg cells, the level of MDSCs was also lower and comparable to control subjects. Our previous study in the group of children with THI showed that up-regulation of IL-12 secretion was associated with hypogammaglobulinaemia. Moreover, during normalisation of IgG level, a decrease of IL-12 level was also observed in these patients [42]. In this context, we hypothesise that IL-12 could play a role in the reduction of MDSCs level in THI patients during their recovery. Although we do not have formal proof, we suggest the likely pathomechanism of THI, based on the positive correlation between the levels of MDSCs and Treg cells (Fig. 6). This hypothesis, however, needs to be further verified with functional assays.

We are aware that the group of patients in our study was small, but because THI is a disease with rather rare frequency, it would be difficult to collect a sufficiently large group of patients in a reasonable timeframe. Moreover, due to ethical reasons, our study has obvious limitations and should be regarded with caution; however, the presented positive correlation between the level of Treg

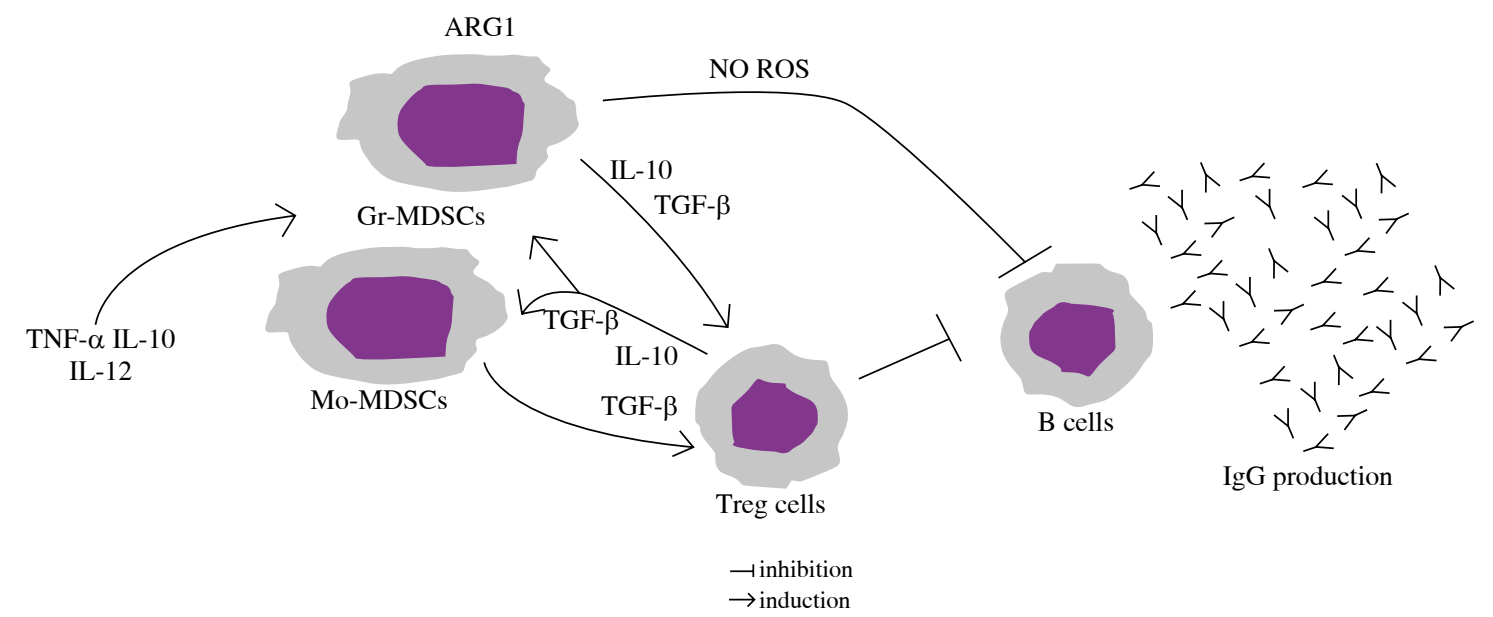

Fig. 6. Simplified scheme of the proposed interactions between MDSCs, Treg cells, and B lymphocytes in THI 
cells and MDSCs suggests a crucial role played by these cells in THI.

\section{Acknowledgments}

This was supported by the Jagiellonian University Collegium Medicum (grant no. K/DSC/003113 and $\mathrm{K} / \mathrm{ZDS} / 005492)$. This article is based upon work from the COST Action BM1404 Mye-EUNITER (http://www. mye-euniter.eu, http://www.mye-euniter.eu), supported by COST (European Cooperation in Science and Technology).

The authors declare no conflict of interest.

\section{References}

1. Gabrilovich DI, Nagaraj S (2009): Myeloid-derived suppressor cells as regulators of the immune system. Nat Rev Immunol 9: 162-174.

2. Zamarron BF, Chen W (2011): Dual roles of immune cells and their factors in cancer development and progression. Int J Biol Sci 7: 651-658.

3. Marvel D, Gabrilovich DI (2015): Myeloid-derived suppressor cells in the tumor microenvironment: expect the unexpected. J Clin Invest 125: 3356-3364.

4. Bronte V, Brandau S, Chen S, et al. (2016): Recommendations for myeloid-derived suppressor cell nomenclature and characterization standards. Nat Commun 7: 1-10.

5. Dumitru CA, Lang S, Brandau S (2013): Modulation of neutrophil granulocytes in the tumor microenvironment: Mechanisms and consequences for tumor progression. Semin Cancer Biol 23: 141-148.

6. Brandau S, Trellakis S, Bruderek K, et al. (2011): Myeloid-derived suppressor cells in the peripheral blood of cancer patients contain a subset of immature neutrophils with impaired migratory properties. J Leukoc Biol 89: 311-317.

7. Pan PY, Li Q, Sato AI, et al. (2006): Gr-1+ CD115+ Immature Myeloid Suppressor Cells Mediate the Development of Tumor-Induced T Regulatory Cells and T-Cell Anergy in Tumor-Bearing Host. Cancer Res 66: 1123-1131.

8. Fujimura T, Kambayashi Y, Aiba S (2012): Crosstalk between regulatory $\mathrm{T}$ cells (Tregs) and myeloid derived suppressor cells (MDSCs) during melanoma growth. Oncoimmunology 1: 1433-1434.

9. Pan PY, Ma Y, Weber KJ, et al. (2010): Immune Stimulatory Receptor CD40 Is Required for T-Cell Suppression and T Regulatory Cell Activation Mediated by Myeloid-Derived Suppressor Cells in Cancer. Cancer Res 70: 99-108.

10. Zea AH, Rodriguez PC, Culotta KS, et al. (2004): L-Arginine modulates $\mathrm{CD} 3 \zeta$ expression and $\mathrm{T}$ cell function in activated human T lymphocytes. Cell Immunol 323: 21-31.

11. Schmielau J, Finn OJ (2001): Activated Granulocytes and Granulocyte-derived Hydrogen Peroxide Are the Underlying Mechanism of Suppression of T-Cell Function in Advanced Cancer Patients. Cancer Res 61: 4756-4760.

12. Youn JI, Gabrilovich DJ (2010): The biology of myeloid derived suppressor cells: the blessing and the curse of morphological and functional heterogeneity. Eur J Imuunol 40: 2969-2975.
13. Haverkamp JM, Smith AM, Weinlich R, et al. (2014): Myeloid-derived suppressor activity is mediated by monocytic lineages maintained by continuous inhibition of extrinsic and intrinsic death pathways. Immunity 41: 947-959.

14. Kumar V, Patel S, Tcyganov E, et al. (2016): The nature of myeloid-derived suppressor cells in the tumor microenvironment. Trends Immunol 37: 208-220.

15. Solito S, Marigo I, Pinton L, et al. (2014): Myeloid-derived suppressor cell heterogeneity in human cancers. Ann N Y Acad Sci 1319: 47-65.

16. Brandau S, Moses K, Lang S (2013): Seminars in Cancer Biology The kinship of neutrophils and granulocytic myeloid-derived suppressor cells in cancer : Cousins, siblings or twins ?. Semin Cancer Biol 23: 171-182.

17. Ren JP, Zhao J, Dai J, et al. (2016): Hepatitis C virus-induced myeloid-derived suppressor cells regulate $\mathrm{T}$-cell differentiation and function via the signal transducer and activator of transcription 3 pathway. Immunology 148: 377-386.

18. Ost M, Singh A, Peschel A, et al. (2016): Myeloid-Derived Suppressor Cells in Bacterial Infections. Front Cell Infect Microbiol 6: 37.

19. Gońi O, Alcaide P, Fresno M (2002): Immunosuppression during acute Trypanosoma cruzi infection: involvement of Ly6G (Gr1(+)CD11b(+) immature myeloid suppressor cells. Int Immunol 14: 1125-1134.

20. Makarenkova VP, Bansal V, Matta BM, et al. (2006): CD11b+/Gr-1+ myeloid suppressor cells cause T cell dysfunction after traumatic stress. J Immunol 176: 2085-2094.

21. Han C, Wu T, Na N, et al. (2016): The effect of immunosuppressive drug cyclosporine A on myeloid-derived suppressor cells in transplanted mice. Inflamm Res 65: 679-688.

22. Al-Muhsen S, Alsum Z (2012): Primary immunodeficiency diseases in the Middle East. Ann N Y Acad Sci 1250: 56-61.

23. Bernatowska E (2008): Pierwotne niedobory odporności. Pediatr Pol 83: 697-703.

24. Quinti I, Soresina A, Spadaro G, et al. 2007: Long-Term Follow-Up and Outcome of a Large Cohort of Patients with Common Variable Immunodeficiency. J Clin Immunol 27: 308-316.

25. Conley ME, Notarangelo LD, Etzioni A (1999): Diagnostic Criteria for Primary Immunodeficiencies. Clin Immunol 93: 190-197.

26. Ovadia A, Dalal I (2014): Transient hypogammaglobulinemia of infancy. Lymph Sign J 1: 1-9.

27. Van Winkle RC, Hauck WW, McGeady SJ (2013): Phenotypic Parameters Predict Time to Normalization in Infants with Hypogammaglobulinemia. J Clin Immunol 33: 1336-1340.

28. Rutkowska M, Lenart M, Bukowska-Strakovŕ K, et al. (2011): The number of circulating CD4+ CD25highFoxp3+ T lymphocytes is transiently elevated in the early childhood of transient hypogammaglobulinemia of infancy patients. Clin Immunol 140: 307-310.

29. Rutkowska M, Trzyna E, Lenart M, et al. (2013): The elevated number of circulating regulatory $\mathrm{T}$ cells in patients with transient hypogammaglobulinemia of infancy is not associated with any abnormalities in the genes encoding the TGF- $\beta$ receptors. Clin Immunol 149: 83-85.

30. Dalal I, Roifman CM (2001): Hypogammaglobulinemia of infancy. Immunol Allergy Clin North Am 21: 129-139.

31. Conley ME, Notarangelo LD, Etzioni A (1999): Diagnostic criteria for primary immunodeficiencies. Representing PAGID (Pan-American Group for Immunodeficiency) and 
ESID (European Society for Immunodeficiencies). Clin Immunol 93: 190-197.

32. Lu T, Ramakrishnan R, Altiok S, et al. (2011): Tumor-infiltrating myeloid cells induce tumor cell resistance to cytotoxic $\mathrm{T}$ cells in mice. J Clin Invest 121: 4015-4029.

33. Sinha P, Clements VK, Bunt SK, et al. (2007): Cross-talk between myeloid-derived suppressor cells and macrophages subverts tumor immunity toward a type 2 response. J Immunol 179: 977-983.

34. Serafini P, Mgebroff S, Noonan K, et al. (2008): Myeloid-Derived Suppressor Cells Promote Cross-Tolerance in B-Cell Lymphoma by Expanding Regulatory T Cells. Cancer Res 68: 5439-5449.

35. Lim HW, Hillsamer P, Banham AH, et al. (2005): Cutting edge: direct suppression of $\mathrm{B}$ - cells by CD4+ CD25+ regulatory T cells. J Immunol 175: 4180-4183.

36. Wang J, Yang L, Yu L, et al. (2017): Surgery-induced monocytic myeloid-derived suppressor cells expand regulatory $\mathrm{T}$ cells in lung cancer. Oncotarget 8: 17050.

37. Hoechst B, Ormandy LA, Ballmaier M, et al. (2008): A new population of myeloid-derived suppressor cells in hepatocellular carcinoma patients induces CD4+ CD25+ Foxp3+ T cells. Gastroenterology 135: 234-243.

38. Lee CR, Kwak Y, Yang T, et al. (2016): Myeloid-derived suppressor cells are controlled by regulatory $\mathrm{T}$ cells via TGF- $\beta$ during murine colitis. Cell Reports 17: 3219-3232.

39. Lelis FJ, Jaufmann J, Singh A, et al. (2017): Myeloid-derived suppressor cells modulate B-cell responses. Immunol Lett 188: 108-115.

40. Meirow Y, Kanterman J, Baniyash M (2015): Paving the road to tumor development and spreading: myeloid-derived suppressor cells are ruling the fate. Front Immunol 6: 523.

41. Kowalczyk D, Mytar B, Zembala M (1997): Cytokine production in transient hypogammaglobulinemia and isolated IgA deficiency. J Allergy Clin Immunol 100: 556-562.

42. Kowalczyk D, Baran J, Webster ADB, et al. (2001): Intracellular cytokine - production by Th1/Th2 lymphocytes and monocytes of children with symptomatic transient hypogammaglobulinemia of infancy (THI) and selective IgA deficiency (SigAD). Clin Exp Immunol 127: 507-512. 